\title{
Has the threat of Zika virus outbreaks come to an end?
}

\author{
Luiz Tadeu Moraes Figueiredo ${ }^{[1]}$
}

[1]. Centro de Pesquisa em Virologia, Faculdade de Medicina de Ribeirão Preto, Universidade de São Paulo, Ribeirão Preto, SP, Brasil.

Zoonotic viruses account for $75 \%$ of emerging infectious diseases in the world. Many of these zoonotic viruses are arthropod-borne viruses (arboviruses) that can be transmitted by mosquitoes, flies, and ticks ${ }^{1}$. One important family of arboviruses is the Flaviviridae, and Zika virus (ZIKV) is a pathogen of the Flavivirus genus. ZIKV is an African virus that causes epidemics of acute febrile illnesses in the tropical world. ZIKV in nature was originally maintained in cycles involving nonhuman primates and mosquitoes of tree canopies, but at some point, the virus adapted to a new cycle involving humans and the anthropophilic mosquito Aedes aegypti. This mosquito lives in cities of tropical countries worldwide and is adapted to urban conditions $^{2}$. Moreover, the sexual transmission of ZIKV after infecting sperm cells has been reported, but it does not seem to be relevant in countries with huge vectorial transmission, such as Brazil. ZIKV, as all Flaviviruses (dengue virus, yellow fever, and West Nile virus), is an enveloped, positive-sense, singlestranded ribonucleic acid (RNA) virus. The last 40 years have shown that Brazil is a suitable destination for the introduction of emergent flaviviruses (dengue, West Nile, ZIKV) ${ }^{3}$.

ZIKV is a common cause of acute benign febrile illness, and when described in Brazil, it was apparently a less important emergent arbovirus compared to dengue and Chikungunya. However, genomic mutations probably increased the virulence of ZIKV. An Asian strain of the virus from Polynesia produced large outbreaks of acute febrile illness with rashes and conjunctivitis in Brazil since 2015. After a couple of months of outbreak, it was reported that ZIKV infections increased the number of cases of Guillain -Barré syndrome, a muscle paralysis syndrome and serious neurological disease caused by autoantibodies that damage the axons of motor neurons because of a viral infection. Furthermore, an increase in the incidence of fetal microcephaly was observed in the northeast region of Brazil, which was soon associated with maternal infection by ZIKV. Congenital disease caused by ZIKV, with microcephaly

Corresponding author: Prof. Luiz Tadeu Moraes Figueiredo e-mail:1tmfigue@fmrp.usp.br

Received 12 June 2018

Accepted 15 June 2018 as a severe manifestation, resulted in an international concern; this led to research efforts for obtaining information on different aspects of the disease. Outbreaks and scientific studies indicate that ZIKV is neurotropic with a particular affinity for neural progenitor cells, leading to extensive destruction of the central nervous system (CNS) during embryogenesis ${ }^{4}$.

Microcephaly is a pathological condition characterized by deficient brain development, and it represents the most dramatic clinical manifestation associated with ZIKV infection. The virus can infect neural progenitor cells and inhibit neurogenesis during brain development. In developing mouse brains, ZIKV infects and replicates in neural progenitor cells, leading to cell cycle arrest, inhibition of cell differentiation, increased cell death, and thinning of the cerebral cortex ${ }^{2}$. It has been shown that ZIKV infects spinal neuroepithelial progenitor cells in vitro and causes disrupted mitosis, structural disorganization, and cell death. Newborns presenting with less severe manifestations of ZIKV congenital disease are increasingly being reported.

During the outbreak in Brazil, it was also found that ZIKV could cause headaches and nuchal rigidity suggestive of meningitis, seizures, mental confusion, or coma in cases of encephalitis and motor dysfunctions in limbs and sphincter dysfunction related to myelitis. ZIKV has caused meningoencephalitis in people of all ages, including immunedepressed patients ${ }^{5}$. Therefore, it is important to consider that an arbovirus requires special capabilities to infect the CNS. The pathogen, besides being virulent, must defeat general cellular and humoral immune responses, be able to cross the bloodbrain barrier (BBB) or the choroid plexus, and when in nervous tissue, defeat the local host defense mechanisms. It is known that flaviviruses bypass the BBB by infecting endothelial cells and releasing their viral progenies on the opposite side, in the CNS for neuroinvasion. Likewise, viruses can infect leukocytes that migrate to the CNS. Innate and adaptive immune system responses could prevent CNS invasion by ZIKV.

Cases of encephalitis by ZIKV and other flaviviruses show a central pattern affecting neurons in the substantia nigra nucleus, thalamus, cerebellum, and cerebral cortex, correlating with neuroradiological findings. These infections, particularly in immunodeficient individuals, can extend to other areas of the brain ${ }^{6}$. 
The serologic diagnosis of ZIKV encounters important difficulties related to the close phylogenetic relation of this virus with dengue viruses, which are also disseminated in Brazil. Thus, conventional serologic assays produce frequent crossreactions in immunoglobulin $\mathrm{G}(\mathrm{IgG})$ and immunoglobulin $\mathrm{M}$ (IgM) antibodies to ZIKV. To avoid cross-reactions with dengue and other flaviviruses, it is important to identify ZIKV-specific antigens for use in serologic assays.

After the 2015-2016 ZIKV outbreak in Brazil, an impressive number of studies on this virus have been performed worldwide. In this edition of the Revista da Sociedade Brasileira de Medicina Tropical (RSBMT), an integrative review on ZIKV publications was conducted in Medline; the data search was performed using the Health Sciences Descriptor Zika Virus. The paper is named Zika virus - a review of the main aspects of this arbovirosis.

Curiously, the circulation of ZIKV has decreased in Brazil during 2017 and 2018, despite the existence of a large number of susceptibles in the native population. However, based on knowledge regarding the cyclic outbreaks of other emergent arboviruses, we know that new outbreaks could occur in the near future after reintroduction of ZIKV from other countries or of viruses that have originated from sylvatic cycles involving other vertebrates and arthropods. Thus, surveillance programs for ZIKV detection, as well as other arboviruses in humans and other animals, must be maintained and encouraged in the country. Furthermore, vaccines are promising for the control of future ZIKV outbreaks, including one that is currently undergoing phase III tests in Brazil.

\section{Conflict of interest}

The author declares that there is no conflict of interest.

\section{REFERENCES}

1. Meltzer E. Arboviruses and viral hemorrhagic fevers (VHF). Infect Dis Clin North Am. 2012;26(2):479-96.

2. Butler D. Zika virus: Brazil's surge in small-headed babies questioned by report. Nature. 2016;530(7588):13-4.

3. Figueiredo LTM. The recent arbovirus disease epidemic in Brazil. Rev Soc Bras Med Trop. 2015;48(3):233-4.

4. Proenca-Modena JL, Milanez GP, Costa ML, Judice CC, Maranhão Costa FT. Zika virus: lessons learned in Brazil. Microbes Infect. 2018;pii:S1286-4579(18)30052-2.

5. Silva IRF, Frontera JA, Filippis AMB, Nascimento OJM, RIO-GBSZIKV Research Group. Neurologic complications associated with the Zika virus in Brazilian adults. JAMA Neurol. 2017;74(10):11908.

6. Schwartzmann PV, Ramalho LNZ, Neder L, Vilar FC, AyubFerreira SM, Romeiro MF, et al. Zika virus associated encephalitis in immunocompromised patient. Mayo Clin Proc. 2017;92(3):460-6. 